\title{
Sub-agent elements for control methods in multi-agent energy management system
}

Conference or Workshop Item

Accepted Version

Yunusov, T., Holderbaum, W. and Potter, B. (2011) Sub-agent elements for control methods in multi-agent energy management system. In: 2011 IEEE 33rd International Telecommunications Energy Conference (INTELEC), 9-13th October 2011, Amsterdam, Netherlands, pp. 1-7. Available at http://centaur.reading.ac.uk/25957/

It is advisable to refer to the publisher's version if you intend to cite from the work. See Guidance on citing.

Published version at: http://dx.doi.org/10.1109/INTLEC.2011.6099780

Publisher: IEEE

All outputs in CentAUR are protected by Intellectual Property Rights law, including copyright law. Copyright and IPR is retained by the creators or other copyright holders. Terms and conditions for use of this material are defined in 
the End User Agreement.

www.reading.ac.uk/centaur

\section{CentAUR}

Central Archive at the University of Reading

Reading's research outputs online 


\title{
Sub-agent elements for control methods in multi-agent energy management system
}

\author{
Timur Yunusov* ${ }^{1}$, Student Member, IEEE; William Holderbaum ${ }^{1}$, Member, IEEE; \\ Ben Potter ${ }^{1}$, Member, IEEE \\ School of System Engineering, University of Reading, Reading, UK ${ }^{1}$ \\ t.yunusov@pgr.reading.ac.uk*
}

\begin{abstract}
Increased penetration of generation and decentralised control are considered to be feasible and effective solution for reducing cost and emissions and hence efficiency associated with power generation and distribution. Distributed generation in combination with the multi-agent technology are perfect candidates for this solution. Pro-active and autonomous nature of multi-agent systems can provide an effective platform for decentralised control whilst improving reliability and flexibility of the grid.

The proposed multi-agent energy management system is aimed at controlling distributed generation and performing demand side management to provide robust and effective grid with lower emissions and costs. Furthermore, appropriate strategy used in combination with the multi-agent energy management system would decrease peak demands in the wider grid. The proposed solution consists of single-type agents who control one or more gird entities which are represented as generic sub-agent elements. The agent applies one control algorithm across all elements and uses cost function to evaluate the suitability of the element as a supplier.

This study shows the ability of the multi-agent energy management system to control grid entities represented by generic sub-agent elements and select suppliers according to the selection behaviour set by the user.
\end{abstract}

Keywords - Multi-agent systems; Smart Grid; Distributed Power Generation; Energy Management.

\section{NOMENCLATURE}

ACL Agent Communication Language

AMS Agent Management system

DF Directory Facilitator

DG Distributed Generation

FIPA Foundation for Intelligent Physical Agents

GUI Graphic User Interface

JADE Java Agent Development framework

MAS Multi-Agent System

LV Low Voltage

$E_{e} \quad$ Element with index $e$

$d_{t, a} \quad$ Estimated demand for agent $a$ at time $t$

$\mathrm{J}_{\mathrm{e}} \quad$ Total cost function for element $\mathrm{e}$

$y_{t, e} \quad$ Output of element $e$ at time $t$

$y_{e}^{\max }$ Maximum output of element $e$

$b_{t, a} \quad$ Supply/demand balance around agent $a$ at time $t$

$c_{t, e} \quad$ Capacity of element $e$ at time $t$

$c_{e}^{\max }$ Maximum capacity of element $e$

$\lambda_{e} \quad$ Priority level for element $e$

$\tau_{t, e} \quad$ Remaining time of operation (duration) for element $e$ at time $t$ $\varepsilon_{e} \quad$ Environmental impact factor for element $e$

$\zeta_{e} \quad$ Price of energy produced by element $e$

$s_{e} \quad$ Operational state of element $e$

$k_{e} \quad$ Availability state of element $e$

$u_{t, e} \quad$ Control signal for element $e$ at time $t$

$\pi_{e} \quad$ Penalty for changing operational state of element $e$

$w_{\zeta} \quad$ Weight for price per watt produced

$w_{\varepsilon} \quad$ Weight for environmental impact factor

$n \quad$ Number of elements in an agent

\section{INTRODUCTION}

C entralised generation and control are prone to losses in transmission and hence, lower efficiency, causing more emissions. Growth in peak demand on the grid also means a greater stress on the transmission network. One of the most popular solutions is to decentralise and increase the penetration of generation across the grid. DG has the potential to improve the efficiency and minimise the cost of energy generation [1]. However, the maximum effect can be achieved only by applying suitable control strategies and methods on DG. Furthermore, a load shifting/shedding strategy coherent with DG control would decrease peak demands in the wider grid and contribute to the reliability of the system.

Multi-agent system is a suitable candidate for controlling DG and performing demand side management, as the nature of the agents is autonomous and pro-active [2] [3]. Indeed, multiagent systems are already taking a major part in the development of a decentralised, robust and effective grid, e.g. by performing power system restoration [4], resource scheduling [5] or power flow control [6].

Current trends in the development of power generating devices show anticipation of a high penetration of distributed micro-generation that will increase the complexity of the distribution network [7]. Therefore, in order to provide flexibility and optimality of multi-agent based control for LV distribution networks, a multi-agent system model with greater flexibility is required.

Recently developed multi-agent solutions are based on different types of agents. These include agents specific for control functions [8] [9] and agents representing each type of entity of the grid (e.g. generators and loads) [10] [11]. This approach requires a separate control strategy for each type of agent, which increases the complexity and cost of the system.

This paper proposes an agent model for representing entities of the grid in a multi-agent system for LV distribution network control. The model represents an entity of the 
network (e.g. a generator, load or storage device) as a single generic element located within an agent - a sub-agent element. This structure would allow more than one entity of a grid to interface with an agent and to apply a single control algorithm across all agents in the LV distribution network.

The rest of the paper is organised as follows: In section 3, the architecture and organisation of the MAS used for this study is presented, including explanation of the sub-agent model and an example of sub-agent elements. Section 4, describes the case study and agents used to evaluate the suitability of sub-agent elements to represent grid entities in the multi-agent energy management system. Section 5 presents the results of the test scenarios. Finally, the paper is ended with sections future work and conclusions.

\section{MULTI-AGENT ENERGY MANAGEMENT SYSTEM}

The purpose of the multi-agent energy management system is to apply decentralised control and optimisation of the grid by the means of autonomous agents, where agent is designed to control an object within the grid. The object can be an entity that has only generating capabilities (e.g. distributed generation station), is an electricity consumer (e.g. a typical household) or is a hybrid of generator and consumer (e.g. household with micro-generation and local storage facilities). The task of the agent is to maintain supply/demand balance within the object whilst minimising operational cost of the elements and trading energy with neighbouring agents. Furthermore, by giving preference to the DG located within
LV distribution network and utilising local storage, agents are able to minimise the peak demand on the main grid and operate in islanded mode if needed. In case when demand exceeds available supply, agent can also apply demand side management by performing load shifting/shedding and hence, reducing the demand.

When agent is searching for the power supplier, the participants of the bidding process are only the neighbouring agents, but the generators, located within the same agent as the load, are also consider as suppliers. If such generator exists within an agent it will have higher chance of being selected as supplier due to smaller transition cost - hence, better efficiency. However, this requires a special structure of the agent.

\section{A. Agent structure}

As mentioned in the introduction, present multi-agent approaches consist of different types of agents for each element. The proposed model of a multi-agent energy management system has only one type of agent, where each entity connected to the agent is represented as a generic element (Figure 1). The agent applies the same control algorithm and operation cost function to all attached elements independently of the actual entity behind the element. The description of an element is given in the next sub-section.

The generic nature of the elements gives the possibility to apply dynamic control strategies on different agents within the network. The user of the agent specifies the type of the control strategy and the agent sets the corresponding weights to the

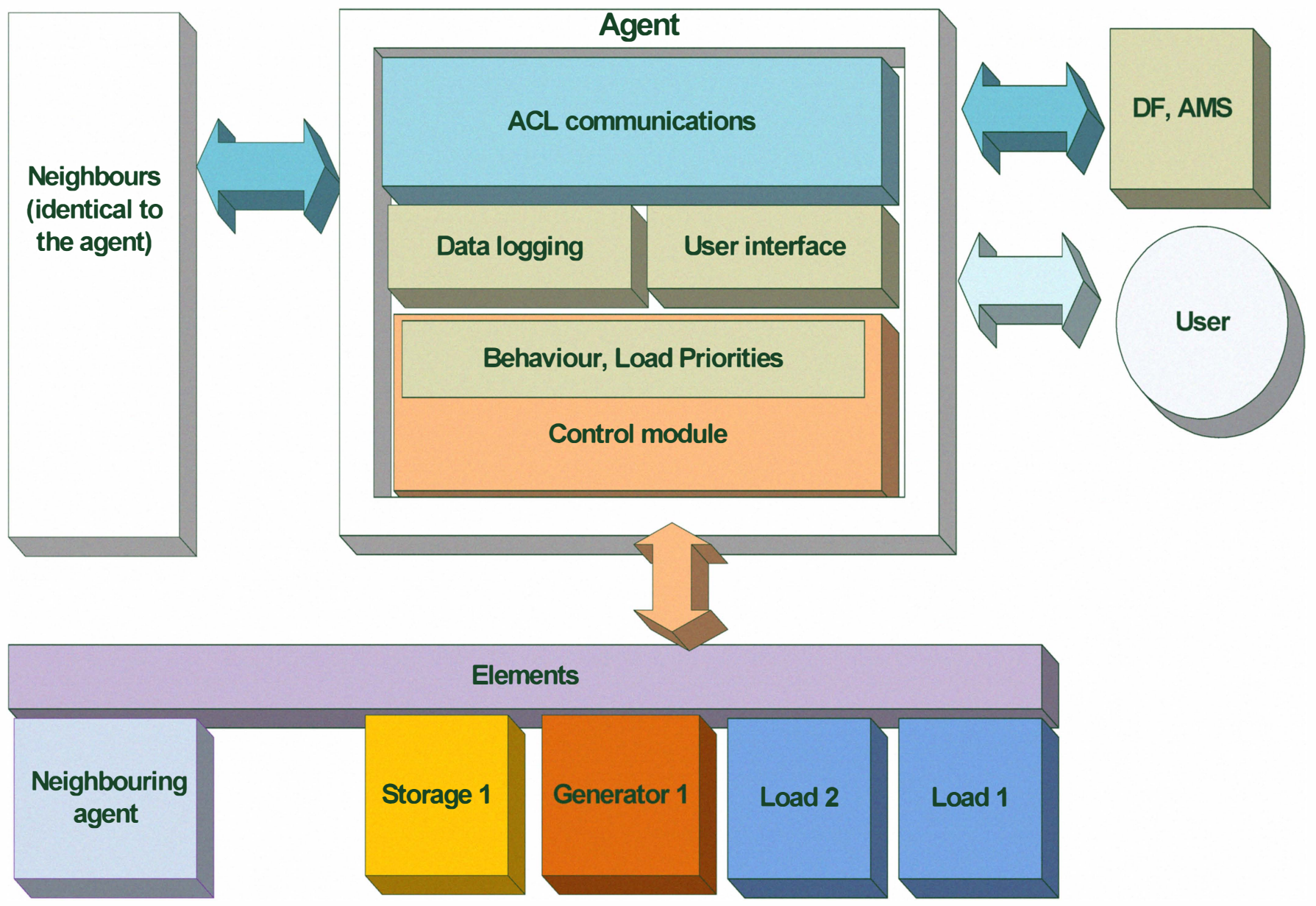

Figure 1. Agent structure 
TABLE I:

ELEMENT PARAMETERS

\begin{tabular}{|c|c|c|}
\hline Parameter & Parameter name & Parameter description \\
\hline $\mathrm{E}$ & Name & Name of the element; (string) \\
\hline $\mathrm{A}$ & Owner & Name of the host agent; (string) \\
\hline $\mathrm{y}_{\mathrm{t}, \mathrm{e}}$ & Output rate $(\mathrm{kW})$ & Current output rate; (double) \\
\hline $\mathrm{y}_{\mathrm{e}}^{\text {max }}$ & Max. output rate $(\mathrm{kW})$ & Maximum output rate; (double) \\
\hline $\mathrm{c}_{\mathrm{t}, \mathrm{e}}$ & Capacity $(\mathrm{kWh})$ & Remaining capacity; (double) \\
\hline $\mathrm{c}_{\mathrm{max}, \mathrm{e}}$ & Max. Capacity $(\mathrm{kWh})$ & Maximum capacity; (double) \\
\hline$\lambda_{\mathrm{e}}$ & Priority level & $\begin{array}{c}\text { Priority level for load shedding/shifting; } \\
\text { O(highest) - "never forced to switch off" to 10(lowest) - "first } \\
\text { to be switched off"; (integer) }\end{array}$ \\
\hline$\tau_{\mathrm{t}, \mathrm{e}}$ & Duration (sec) & Estimated running time; (integer) \\
\hline$\varepsilon_{\mathrm{e}}$ & $\begin{array}{c}\text { Impact factor } \\
\text { (CO2 kg/kWh) }\end{array}$ & $\begin{array}{c}\text { Environmental impact associated with every kW produced; } \\
\text { (double) }\end{array}$ \\
\hline$\zeta_{\mathrm{e}}$ & Cost (p/kWh) & Cost of every watt produced; (double) \\
\hline $\mathrm{s}_{\mathrm{e}}$ & State (on/Off) & Operational state; (Boolean) \\
\hline $\mathrm{k}_{\mathrm{e}}$ & Connected (online/offline) & Availability state; (Boolean) \\
\hline $\mathrm{u}_{\mathrm{t}, \mathrm{e}}$ & Control signal & From -1(charging for battery) to +1; (double) \\
\hline$\pi_{\mathrm{e}}$ & State change penalty & Penalty associated with changing operational state; (integer) \\
\hline
\end{tabular}

parameters of the elements. The weights of the parameters are then used for calculating the suitability cost function for a generator as potential supplier. For example, a generator can be selected as supplier based on its price per $\mathrm{kWh}$ or kilograms of $\mathrm{CO}^{2}$ per $\mathrm{kWh}$ produced.

Initially agent estimates the internal balance, which includes internal consumption and generation as well as power exports and imports to the neighbouring agents. Once the internal balance is negative, the agent evaluates the generating capabilities of internal generators and requesting costs of generation from the neighbours. During the bidding process the agent communicates with neighbours in the LV network to exchange the information regarding power requirements and power availability. Once the initial information exchange is complete, each agent creates a new element which represents the power generating and consuming part of the neighbouring agent. The communication is provided through an ACL module in JADE [12] and is used for social discovery, bidding process and energy supply/demand control. Each agent has control over its own generators and can apply priority load shedding to the attached loads. Agent will export the agreed amount of power as long as it can provide it and will notify the recipient if export is interrupted.

\section{B. Sub-Agent element}

The main purpose of introducing a generic sub-agent element is to make an agent versatile in interfacing to various grid entities and applying the same control algorithm to all attached elements. Therefore, the parameters of an element must be common for major types of entities in the grid. The parameters of an element and their description are given in Table 1.
Depending on the functional nature of the entity, the element will have specific parameter values that describe its function. The power output is positive for a generator and negative for a load. Similarly, the range of values for a parameter of an element is dependent on the type of entity. A regulated generator has a control signal varying from zero to maximum output, where a fixed output generator or a load has ON or OFF control signal.

The capacity of the element is mostly used to determine the most suitable energy source; a local storage device with very low power capacity is unlikely to be selected as a suitable supplier. Most types of generators can have a near infinite capacity, given the fuel is provided on time. However, for some renewable power sources, the capacity term could provide the predicted availability based on, for example, the forecasted wind speed for wind turbines or remaining daylight time for photo-voltaic cell. The list of parameters can be extended to accommodate new entities of the grid and new parameters can be easily mapped to the existing entities. Furthermore, additional parameters can be added to the subagent element in order to apply a new control strategy that requires additional information about the elements (e.g. reactive power control, scheduling or applying social factors)

\section{System model and cost function for a sub-agent element}

Since the purpose of this study is to evaluate the feasibility of using generic elements for multi-agent control of grid entities, the model representing these entities is assumed to be a perfect input/output system. The model has the following form:

$$
\mathrm{y}_{\mathrm{t}, \mathrm{e}}=\mathrm{u}_{\mathrm{t}, \mathrm{e}} \cdot \mathrm{y}_{\mathrm{e}}^{\max }
$$

Where 


$$
\begin{gathered}
\mathrm{y}_{\mathrm{t}, \mathrm{e}}, \mathrm{y}_{\text {max }, \mathrm{e}} \in[-1000000 ; 1000000] \subset \mathbb{R} ; \\
\mathrm{u}_{\mathrm{t}, \mathrm{e}} \in[-1,1] ; \\
\mathrm{e} \in\{1,2,3 . . \mathrm{n}\}, \quad \mathrm{t} \in \mathbb{R}
\end{gathered}
$$

The total supply/demand around agent is a sum of outputs of all elements:

$$
\mathrm{b}_{\mathrm{t}, \mathrm{a}}=\sum_{\mathrm{e}=0}^{\mathrm{n}} \mathrm{y}_{\mathrm{t}, \mathrm{e}}
$$

When $b_{t, a}$ reaches negative values, agent checks generating capabilities of the attached elements (attached generators, batteries and neighbouring agents). The fitness of an element as a potential supplier depends on its current parameters and the rate of requested power and evaluated using following cost function:

$$
\begin{gathered}
\mathrm{J}_{\mathrm{e}}\left(\mathrm{E}_{\mathrm{e}}, \mathrm{d}_{\mathrm{t}}\right)=\mathrm{e}^{\left(\frac{2 \mathrm{~d}_{\mathrm{t}}}{c_{\max }}+\frac{10}{c_{\mathrm{t}, \mathrm{e}}}\right)}+\mathrm{e}^{\left(\frac{-10\left(\mathrm{~d}_{\mathrm{t}}+\mathrm{y}_{\mathrm{t}}\right)}{y_{\max }}\left(\mathrm{ymax}_{\max }-\mathrm{y}_{\mathrm{t}}-\mathrm{d}_{\mathrm{t}}\right)\right)}+ \\
\mathrm{e}^{-10 \mathrm{y}_{\max }}+\varepsilon_{\mathrm{e}} \cdot \mathrm{w}_{\varepsilon}+\zeta_{\mathrm{e}} \cdot \mathrm{w}_{\zeta}
\end{gathered}
$$

Where

$$
\mathrm{J}_{\mathrm{e}}\left(\mathrm{E}_{\mathrm{e}}, \mathrm{d}_{\mathrm{t}}\right)=\mathrm{J}_{\mathrm{e}}\left(\left\{\mathrm{s}_{\mathrm{e}}, \pi_{\mathrm{e}}, \mathrm{y}_{\mathrm{t}, \mathrm{e}}, \mathrm{c}_{\mathrm{t} . \mathrm{e}}, \mathrm{k}_{\mathrm{e}}, \varepsilon_{\mathrm{e}}, \zeta_{\mathrm{e}}\right\}, \mathrm{d}\right)
$$

The price per $\mathrm{kWh}, \zeta_{\mathrm{e}}$, and impact factor (measured in $\mathrm{kg}$ of $\mathrm{CO}^{2}$ per $\left.\mathrm{kWh}\right), \varepsilon_{\mathrm{e}}$ are weighted according to the behaviour set by the user. Currently there are three behaviour options: "normal", "cheap" and "green". For the "normal" behaviour the weights for the parameters are normalised and produce equal effect on the cost function. For the other two behaviours the appropriate parameter has a greater weight in order to increase its effect on the cost function. Once the costs for all available elements are calculated, the best supplier element is given by:

$$
\mathrm{e}_{\mathrm{s}}=\arg \min _{\mathrm{e} \in\{0,1,2, \ldots, \mathrm{n}\}}\left(\mathrm{J}_{\mathrm{e}}\left(\mathrm{E}_{\mathrm{e}}, \mathrm{d}\right)\right)
$$

If the supply/demand balance remained negative after all available generators are operational, the agent will perform load shedding function and switch off load with the lowest priority. When supply exceeds the demand the output of the least suitable supplier is reduced until $\beta_{t, a}$ is zero. This applies to the internal sources as well as neighbouring agents. With external sources the agents sends an action request to the supplying neighbour which then makes appropriate control adjustments.

\section{Implementation}

The multi-agent energy management system is implemented using JADE. The framework is designed to simplify the implementation and development of multi-agent systems by providing a software platform that complies with FIPA specifications [13]. JADE also provides tools for debugging and deployment phases and supports ACL as the basis for agent communication architecture.

The agent consists of two main components: GUI and agent's body. The purpose of the GUI (see figures 2 and 3 ) is to provide control over the elements attached to the agent, set the supplier selection behaviour and monitor the power exchange with neighbours as well as other statistics. The body of the agent deals with all of the communications, servicing elements and maintaining book keeping.

Thanks to the ACL module in JADE communications are easy to handle and requires only performing appropriate actions to informative and content of the message. Since all the agents are identical in structure, every message that agent sends it must produce the appropriate reply and consequently process the reply.

Servicing of the elements includes estimating the demand and generating capabilities within the agent and complying with the element model when operating an element (i.e. current output does not exceed maximum output, load does not operate beyond specified duration period etc)

Book keeping maintains the record of the major actions of the agent. These include: changing operational states of the

TABLE II:

INITIAL CONDITIONS FOR THE ELEMENTS USED IN THE CASE STUDY

\begin{tabular}{|c|c|c|c|c|}
\hline Parameter & Element 0 & Element 1 & Element 2 & Element 3 \\
\hline$E$ & Electric motor & Freezer & Gas turbine & Pico hydropower \\
\hline A & Alpha & Alpha & Beta & Beta \\
\hline$y_{t, e}$ & 0 & 0 & 0 & 0 \\
\hline$y_{\max , \boldsymbol{e}}$ & -10 & -4.5 & 20 & 20 \\
\hline$c_{t, e}$ & 0 & 0 & INF & INF \\
\hline$c_{\text {max }, e}$ & 0 & 0 & INF & INF \\
\hline$\lambda_{e}$ & 4 & 3 & 0 & 0 \\
\hline$\tau_{t, e}$ & $\mathrm{INF}$ & $\mathrm{INF}$ & $\mathrm{INF}$ & INF \\
\hline$\varepsilon_{e}$ & 0 & 0 & 12 & 20 \\
\hline$\zeta_{e}$ & 0 & 0 & 0.545 & 0.2 \\
\hline$s_{e}$ & 1 & 1 & 0 & 1 \\
\hline$k_{e}$ & 1 & 1 & 1 & 0 \\
\hline$u_{t, e}$ & 0 & 0 & 0 & 20 \\
\hline$\pi_{e}$ & 0 & 0 & 20 & \\
\hline
\end{tabular}


elements, negotiations with neighbours and power imported or exported to which neighbour.

\section{CASE STUDY}

In order to test the feasibility of the proposed multi-agent energy management system, there were created two agents (Alpha and Beta) with a number of attached elements. The parameters of the attached elements are given in table ii.

The load elements on Alpha are operating and consuming $14.5 \mathrm{~kW}$. Since Alpha does not have any generating capability, it has to request its neighbour Beta to supply the required amount of power. Table ii shows that Beta has two elements with generating capabilities but with different values for price and impact factor.

There are three simulation periods each starting with both loads on Alpha being switched on. However the supplier selection behaviour will be changed for each simulation period, first "normal, then "cheap" and finally "green".

\section{RESULTS}

The multi-agent energy management system was tested on the feasibility of using sub-agent element models to represent typical grid entities and using simple cost function to select suitable supplier for three simulation periods.

Table ii shows how two types of entities (load and generator) were represented as sub-agent elements. One of the major differences between two types is the polarity of the output (negative output for loads and positive for generators). Battery type entities are represented by elements with positive outputs and maximum capacity less than infinity.

Figure 3 presents the GUI of the agent Beta which supplies $14.5 \mathrm{~kW}$ to the neighbouring agent Alpha. Figure 3 shows the GUI for the gent Alpha which houses the operating loads and

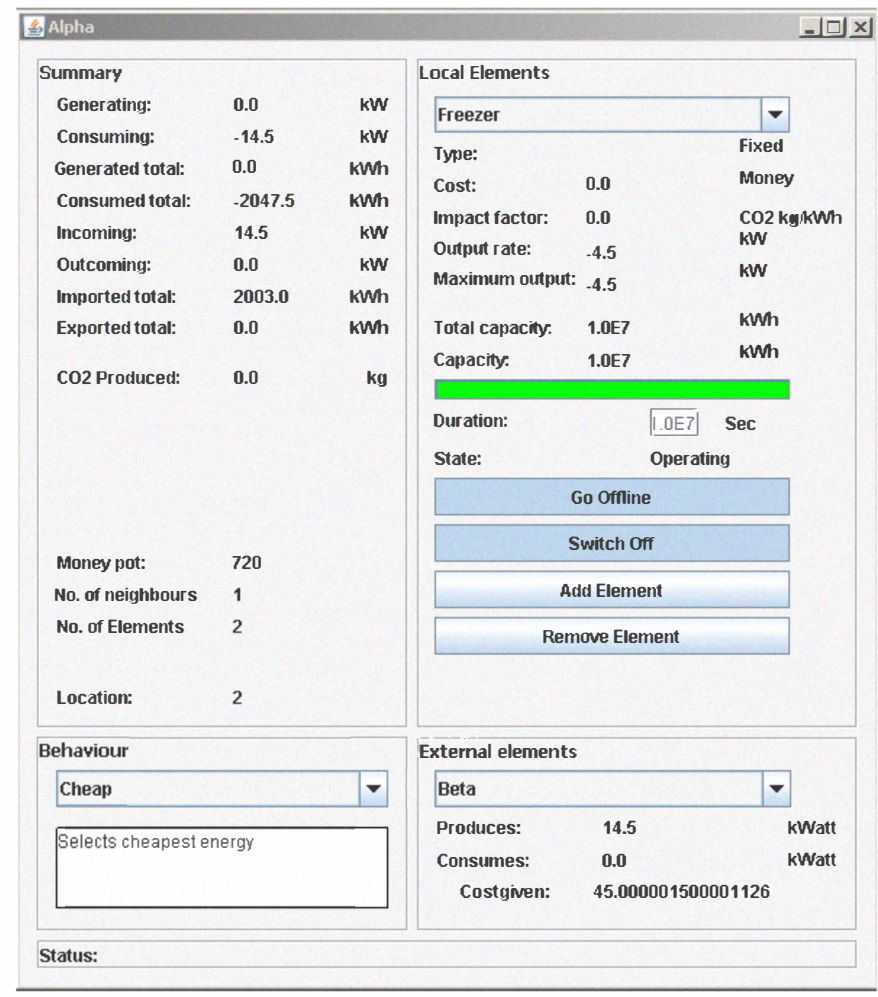

Figure 2. GUI for monitoring and managing agent Alpha.
TABLE III:

TOTAL COSTS $\mathrm{J}_{\mathrm{E}}$ EVALUATED FOR DIFFERENT SELECTION BEHAVIOURS

\begin{tabular}{|l|l|l|l|}
\hline & Normal & Cheap & Green \\
\hline Element 2 & 87 & 45 & 75 \\
\hline Element 3 & 41 & 61 & 21 \\
\hline
\end{tabular}

imports all required power from Beta. Once a second, Alpha estimates the internal balance, which will be negative at the start of each simulation period and requests Beta to provide cost for supplying $14.5 \mathrm{~kW}$. This stage can be seen on figure 4 which shows the screenshot of the output console for JADE during the simulation period with selection behaviour set to "Cheap". Beta evaluates cost of each attached element to generate $14.5 \mathrm{~kW}$ and the smallest calculated cost is sent to Alpha in response to the request for power and Alpha will select the supplying agent with the smallest cost out of all responded agents. Since there are only two agents in the simulation and only one of them with generating capability, Alpha will choose Beta as the supplier as long as the quoted costs are below the limit of 300 . If the quoted costs are above the limit then there are no suitable suppliers and Alpha will apply load shedding to reduce demand.

The costs calculated at Beta for each of the simulation period are given in table iii. The costs for elements located at Beta correspond to the given selection behaviour. For the "normal" behaviour element " 2 " will have higher cost since the difference in prices is practically negligible, but the impact factor for element " 3 " is significantly smaller. Therefore the preferred supplier is element " 3 " - pico-hydropower generator. From the table ii and figure 4 it can be seen that Beta selects element "2" (gas turbine generator) as the supplier with smallest price when the selection behaviour is set to "cheap" option.

Once Alpha accepts Beta as supplier, Beta activates the

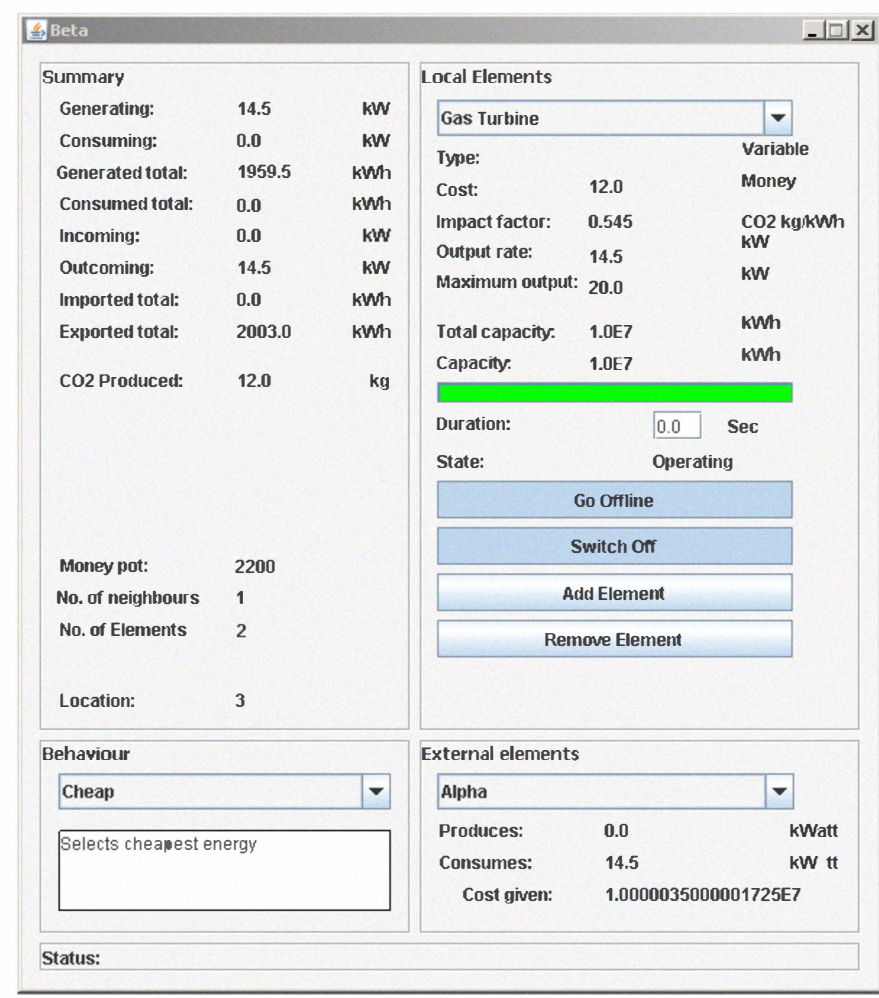

Figure 3. GUI for monitoring and managing agent Beta. 
Alpha@[...]JADE Element Freezer has output of -4.5

Alpha@[...]JADE Element Electric motor has output of -10.0

Alpha@[...]JADE Neighbour Beta supplies 0.0 and consumes 0.0

Alpha@[...]JADE Pre-control balance is -14.5 , required power is -14.5 , supply is: 0.0

Alpha@[...]JADE Element Freezer has a cost of 3.4934271057485095E19 for demand of 14.5

Alpha@[...]JADE Element Electric motor has a cost of 2.6881171418161356E43 for demand of 14.5

Alpha@[...]JADE Element Freezer has output of -4.5

Alpha@[...]JADE Element Electric motor has output of -10.0

Alpha@[...]JADE Neighbour Beta supplies 0.0 and consumes 0.0

Alpha@[...]JADE Post-control balance is -14.5 , demand is -14.5 , supply is: 0.0

Alpha@[...]JADE Requesting cost from neighbours

Beta@[...]JADE Element Gas Turbine has a cost of 45.000001500001126 for demand of 14.5

Beta@[...]JADE Element Pico Hydro has a cost of 61.00000172500149 for demand of 14.5

Beta@[...]JADE Neighbour Alpha@134.225.205.123:1099/JADE requests 14.5

Alpha@[...]JADE Neighbour (agent-identifier :name Beta@[...]JADE :addresses ([...] )) has cost 45.000001500001126

Alpha@[...]JADE Sending control command to neighbour Beta@[...]/JADE

Beta@[...]JADE Applying internal control on Gas Turbine to output 14.5 control signal is: 0.725 and output is: 14.5

Alpha@[...]JADE Neighbour (agent-identifier :name Beta@[...]JADE :addresses [...])) supplies us 14.5

...

Beta@[...]JADE Element Gas Turbine has output of 14.5

Beta@[...]JADE Element Pico Hydro has output of 0.0

Beta@[...]ADE Neighbour Alpha supplies 0.0 and consumes 14.5

Beta@[...]JADE Pre-control balance is 0.0 , required power is 0.0 , supply is: 14.5

Beta@[...]JADE Element Gas Turbine has output of 14.5

Beta@[...]JADE Element Pico Hydro has output of 0.0

Beta@[...]JADE Neighbour Alpha supplies 0.0 and consumes 14.5

Beta@[...]JADE Post-control balance is 0.0 , demand is -14.5 , supply is: 14.5

Alpha@[...]JADE Element Freezer has output of -4.5

Alpha@[...]JADE Element Electric motor has output of -10.0

Alpha@[...]JADE Neighbour Beta supplies 14.5 and consumes0.0

Alpha@[...]JADE Pre-control balance is 0.0 , required power is 0.0 , supply is: 14.5

Alpha@[...]JADE Element Freezer has output of -4.5

Alpha@[...]JADE Element Electric motor has output of -10.0

Alpha@[...]JADE Neighbour Beta supplies 14.5 and consumes0.0

Alpha@[...]JADE Post-control balance is 0.0 , demand is -14.5, supply is: 14.5

Figure 4. Screenshot of the output console for JADE.

selected element to supply power and exports it to Alpha (see figure 4).

\section{FUTURE WORK}

Currently, the multi-agent energy management system operates on a simple heuristic algorithm. The future plan is to improve the algorithm to handle change in availability of suppliers and change in their parameters. The improved cost function will include physical parameters specific to the element operating within agent and will have improved adjustment to the behaviour set by the users. Furthermore, optimisation algorithms will be introduced to improve the utilisation of available generation and storage facilities.

\section{CONCLUSION}

This paper presented a novel multi-agent energy management system aimed at optimising the usage of the grid through decentralised control of the grid entities (e.g. loads, generators, batteries) whilst improving the reliability and flexibility of the grid. All agents in the energy management system are identical in their structure and are in control of the attached entities. The grid entities are represented as sub-agent elements and the parameters of the element define the operational nature of the grid entity represented by this element. The parameter of the element can be extended to accommodate new grid entities or provide entity specific information required for advanced control algorithms.

A case study demonstrated the structure of the agent controlling attached grid entities. Internal structure of the agent was presented along with the GUI for monitoring agent and managing elements. Different types of grid entities were represented as sub-agent elements and a cost function was used to evaluate their suitability as a supplier under theuser defined preferences. The multi-agent energy management system selected the supplying elements according to the set selection behaviour.

\section{REFERENCES}

[1] L.M. Tolbert and F.Z. Peng, "Scalable multi-agent system for real-time electric power management," 2001 Power Engineering Society Summer 
Meeting. Conference Proceedings (Cat. No.01CH37262), vol. 00, 2001, pp. 1676-1679.

[2] S.D.J. McArthur, E.M. Davidson, V.M. Catterson, A.L. Dimeas, N.D. Hatziargyriou, F. Ponci, and T. Funabashi, "Multi-Agent Systems for Power Engineering Applications-Part I: Concepts, Approaches, and Technical Challenges," IEEE Transactions on Power Systems, vol. 22, Nov. 2007, pp. 1743-1752.

[3] S.D.J. McArthur, E.M. Davidson, V.M. Catterson, A.L. Dimeas, N.D. Hatziargyriou, F. Ponci, and T. Funabashi, "Multi-Agent Systems for Power Engineering Applications-Part II: Technologies, Standards, and Tools for Building Multi-agent Systems," IEEE Transactions on Power Systems, vol. 22, Nov. 2007, pp. 1753-1759.

[4] T. Nagata and H. Sasaki, "A multi-agent approach to power system restoration," IEEE Transactions on Power Systems, vol. 17, May. 2002, pp. 457-462.

[5] T. Logenthiran, D. Srinivasan, and A.M. Khambadkone, "Multi-agent system for energy resource scheduling of integrated microgrids in a distributed system," Electric Power Systems Research, vol. 81, Jan. 2011, pp. 138-148.

[6] P.H. Nguyen, W.L. Kling, G. Georgiadis, M. Papatriantafilou, and L. Bertling, "Distributed routing algorithms to manage power flow in agent-based active distribution network," Innovative Smart Grid Technologies Conference Europe (ISGT Europe), 2010 IEEE PES, IEEE, 2010, p. 1-7.

[7] F. van Overbeeke, "Active networks: Distribution networks facilitating integration of distributed generation," 2nd International Symposium on Distributed Generation.

[8] L.M. Tolbert and FZ. Peng, "Scalable multi-agent system for real-time electric power management," 2001 Power Engineering Society Summer Meeting. Conference Proceedings (Cat. No.01CH37262), vol. 00, 2001, pp. 1676-1679.

[9] Heussen, K.; Saleem, A.; Lind, M.; , "System-awareness for agent-based power system control," Bulk Power System Dynamics and Control (iREP) - VIII (iREP), 2010 iREP Symposium, vol., no., pp.1-15, 1-6 Aug. 2010

[10] T. Logenthiran, D. Srinivasan, and D. Wong, "Multi-agent coordination for DER in MicroGrid," 2008 IEEE International Conference on Sustainable Energy Technologies, Nov. 2008, pp. 77-82.

[11] H. Fakham, A. Ahmidi, F. Colas, and X. Guillaud, "Multi-agent system for distributed voltage regulation of wind generators connected to distribution network," Innovative Smart Grid Technologies Conference Europe (ISGT Europe), 2010 IEEE PES, IEEE, , p. 1-6.

[12] JADE, Available : http://jade.tilab.com

[13] FIPA standards, Available: http://www.fipa.org/ 